\title{
MICROWAVE ASSISTED SYNTHESIS OF 2-ARYL BENZIMIDAZOLE
}

\author{
Ajay Kharche*, Sandeep Waghulde, Nilesh Gorde, Dr. Mohan Kale
}

Konkan Gyanpeeth Rahul Dharkar College of Pharmacy and Research Institute, Karjat, Dist: Raigad, Maharashtra, India

\begin{abstract}
:
Various 2-aryl benzimidazole derivatives were synthesized using Microwave assisted reaction. The solvent and catalyst were selected to get product with good yield. From ophenylenediamine and different substituted aromatic carboxylic acid using ethyl acetate as a catalyst derivative of 2-substituted benzimidazole were synthesized. All the synthesized compounds were screened by FTIR and 1H-NMR spectra.
\end{abstract}

Keywords: $o$-phenylenediamine, benzimidazole, Ethyl acetate, Aromatic carboxylic acid.

\section{INTRODUCTION:}

Microwave assisted synthesis is the process of applying microwave radiation to chemical reactions. [1] Microwave assisted synthesis is a major breakthrough in the field of medicinal synthetic chemistry; a drastic change in the way of organic compound synthesis is performed. [2] Conventional synthesis of organic compounds is a lengthy and somehow inconvenient process. [3] Microwave assisted synthesis gives the scientists more time to perform their creativity, also in short period of time scientists can develop various theories and also possible to develop new processes for the synthesis of organic compounds. [4] In conventional method of synthesis scientists spends several hours or sometime lots of days for the synthesis of organic compounds, but using microwave assisted reaction they can synthesize various compounds in a minute. [5] Conventional method also faces the problem associated with waste disposal of solvents, it can be overcome by performing reactions without a solvent under microwave irradiation. Thus, microwave synthesis acts as a potential tool for green chemistry. [6]

Benzimidazole Pharmacophore are a very important class of the heterocyclic organic chemistry it shows various pharmacological activities [7] viz. antiulcer [8], analgesic [9], antitumor [10], antiviral [11] and anti-HIV [12] etc.

The objective of the present research work was to develop the green and efficient microwave assistant methods to synthesize 2-arylbenzimidazole derivatives from aromatic carboxylic acids and ethyl acetate, and its characterization.

\section{EXPERIMENTAL SECTION:}

All the solvents, chemicals and drugs employed for the synthetic work were of S D Fine/ Qualigens/ E. Merck/ Laboratory grade.

Melting point was uncorrected. Purity of the compounds was ascertained by precoated TLC plates (E. Merck Kieselgel 60 F254, Mumbai, India).

\section{MATERIALS \& METHODS:}

It had been planned to synthesize compounds containing 2-aryl benzimidazole. 


\section{PROCEDURE:}

O-phenylenediamine (0.01 moles) with aromatic carboxylic acid (0.01 moles), ethyl acetate $(0.0025$ mole $)$ in water $(15 \mathrm{ml})$ were irradiated in microwave at $90 \%$ power $(765 \mathrm{~W})$. After completion of reaction, the reaction mixture was cooled to room temperature and made just alkaline to litmus by slowly adding $10 \% \mathrm{NaOH}$. The product was filtered and washed with cold water, recrystallized with absolute ethanol. The conventional synthesis of 2-aryl benzimidazole were performed and compared with microwave assisted synthesis method. It was found that the reaction time was comparatively less from $\mathrm{hr}$ to min and the $\%$ yield were found to be high when compared to conventional method. Above synthesis required a conventional reaction time of 2-15 hours while the yields were always poor ( $>50 \%)$ therefore it was felt worthwhile to study these reactions under microwave induced technique with the aim of decreasing the reaction time and increasing the yield. The five derivatives were synthesized using different aromatic carboxylic acids. (Table I). The completion of the reaction was monitored by TLC ethanol: water $(8: 2)$.

\section{ROUTE OF SYNTHESIS:}<smiles>Nc1ccccc1N</smiles>

o-Phenylene diamine<smiles>[R]C(=O)O</smiles>

Carboxylic acid<smiles>[R]c1nc2ccccc2[nH]1</smiles>

2-aryl benzimidazole

$\mathrm{R}=$ Phenyl, Cinnamyl, 2-chloro phenyl, 3-nitro phenyl, 3,5-dinitro salicylyl

Table I: 2-arylbenzimidazole derivatives

\begin{tabular}{|l|c|c|c|}
\hline Compound & $\begin{array}{c}\text { Reaction Time } \\
\text { in min. (h) }\end{array}$ & $\begin{array}{c}\text { Percentage Yield } \\
(\%)\end{array}$ \\
\hline Ia & & 3.5 & 96 \\
\hline $\mathrm{Ic}$ & & \\
\hline
\end{tabular}




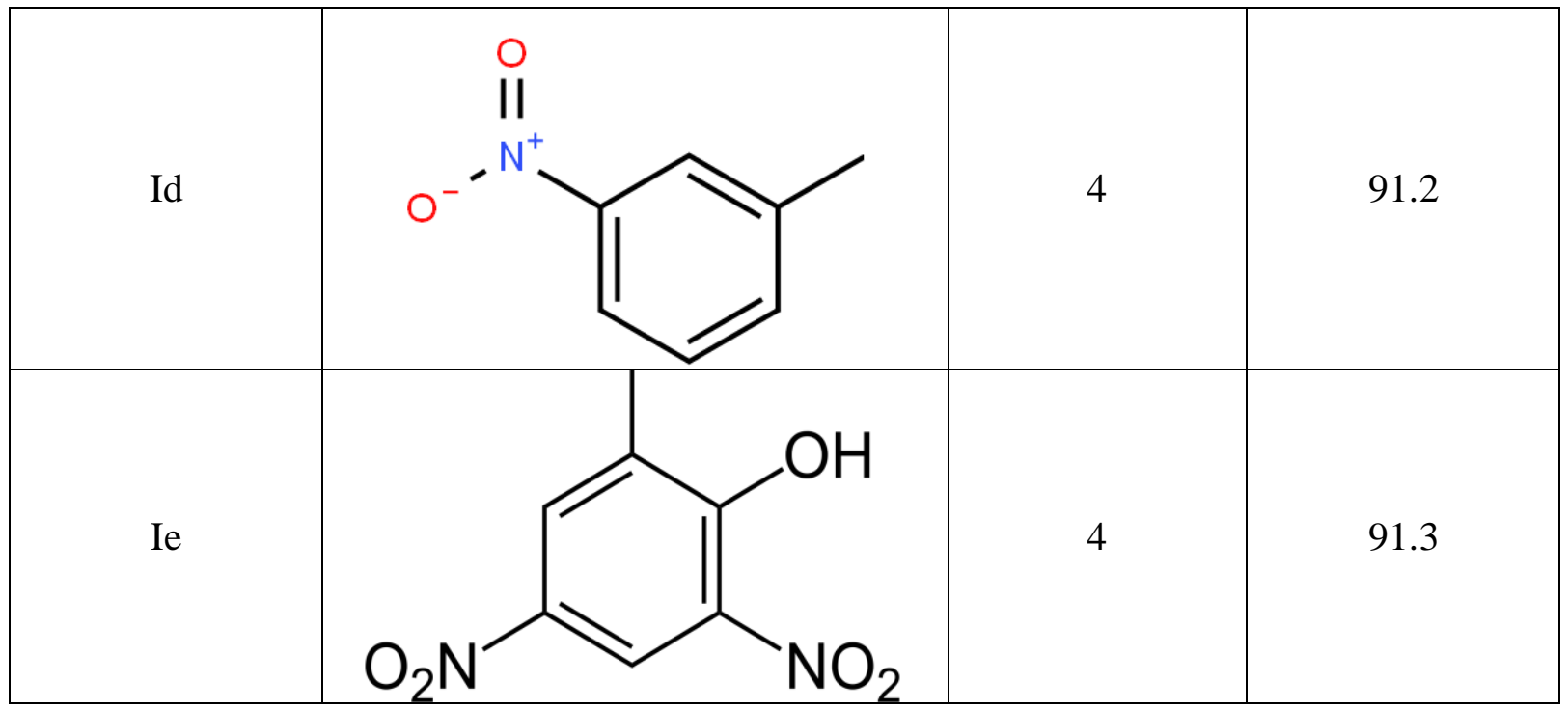

\section{SPECTRAL DATA:}

2-phenyl benzimidazole Ia: White solid. Yield: $96 \%$, m.p. $298-300^{\circ} \mathrm{c}$

${ }^{1} \mathrm{H}$ NMR: $\delta 7.06(1 \mathrm{H}$, ddd, $J=7.7,7.6,1.3 \mathrm{~Hz}), 7.17(1 \mathrm{H}$, ddd, $J=8.1,7.6,1.6 \mathrm{~Hz}), 7.42-$ $7.51(3 \mathrm{H}, 7.47$ (dddd, $J=7.7,7.5,1.2,0.4 \mathrm{~Hz}), 7.47(\mathrm{tdd}, J=7.5,1.5,1.4 \mathrm{~Hz})$ ), 7.87-7.93 $(2 \mathrm{H}, 7.90(\mathrm{ddd}, J=7.7,1.6,0.5 \mathrm{~Hz}), 7.90(\mathrm{ddd}, J=8.1,1.3,0.5 \mathrm{~Hz})), 8.10(2 \mathrm{H}$, dddd, $J=$ $7.7,1.5,1.4,0.4 \mathrm{~Hz})$.

2-cinnamyl benzimidazole Ib : Light Brown. Yield: 92.8\%, m.p. 204-207 $\mathrm{c}$

${ }^{1} \mathrm{H}$ NMR: $\delta 7.06(1 \mathrm{H}, \mathrm{ddd}, J=7.7,7.5,1.3 \mathrm{~Hz}), 7.13-7.23(2 \mathrm{H}, 7.19(\mathrm{~d}, J=13.7 \mathrm{~Hz}), 7.17$ $(\mathrm{ddd}, J=8.1,7.5,1.4 \mathrm{~Hz})$ ), 7.26-7.46 (6H, 7.37 (dddd, $J=8.1,7.6,1.5,1.4 \mathrm{~Hz}), 7.42(\mathrm{~d}, J=$ $13.7 \mathrm{~Hz}), 7.42$ (dddd, $J=8.1,1.8,1.5,0.5 \mathrm{~Hz}), 7.31(\mathrm{tdd}, J=8.1,1.6,0.5 \mathrm{~Hz})), 7.61(1 \mathrm{H}$, ddd, $J=8.1,1.3,0.4 \mathrm{~Hz}), 7.85(1 \mathrm{H}$, ddd, $J=7.7,1.4,0.4 \mathrm{~Hz})$.

2-Chlorophenyl benzimidazole Ic : Light Yellow. Yield: 89\%, m.p. 239-243 ${ }^{\circ} \mathrm{c}$

${ }^{1} \mathrm{H}$ NMR: $\delta$ 7.10-7.20 (2H, 7.14 (ddd, $\left.J=7.7,7.6,1.4 \mathrm{~Hz}\right), 7.15(\mathrm{ddd}, J=8.1,7.6,1.6 \mathrm{~Hz})$ ), 7.45-7.57 (2H, 7.50 (ddd, $J=7.7,7.6,1.1 \mathrm{~Hz}), 7.53$ (ddd, $J=8.1,7.7,1.6 \mathrm{~Hz})$ ), $7.74(1 \mathrm{H}$, ddd, $J=8.1,1.1,0.4 \mathrm{~Hz}$ ), 7.83-7.91 (3H, 7.86 (ddd, $J=7.7,1.6,0.5 \mathrm{~Hz}), 7.86$ (ddd, $J=7.6$, 1.6, $0.4 \mathrm{~Hz}), 7.88$ (ddd, $J=8.1,1.4,0.5 \mathrm{~Hz})$ ).

3-nitrophenyl benzimidazole Id : Light Brown. Yield: 91.2\%, m.p. 299-302

${ }^{1} \mathrm{H}$ NMR: $\delta 7.29(1 \mathrm{H}, \mathrm{ddd}, J=8.1,7.5,1.5 \mathrm{~Hz}), 7.54(1 \mathrm{H}, \mathrm{ddd}, J=7.6,7.5,1.5 \mathrm{~Hz}), 7.65$ $(1 \mathrm{H}, \mathrm{ddd}, J=8.6,7.8,0.4 \mathrm{~Hz}), 7.80(1 \mathrm{H}, \mathrm{ddd}, J=8.1,1.5,0.4 \mathrm{~Hz}), 7.98(1 \mathrm{H}$, ddd, $J=7.6$, $1.5,0.4 \mathrm{~Hz}), 8.06(1 \mathrm{H}, \mathrm{dt}, J=7.8,1.5 \mathrm{~Hz}), 8.29(1 \mathrm{H}$, ddd, $J=8.6,1.6,1.5 \mathrm{~Hz}), 8.83(1 \mathrm{H}$, $\mathrm{td}, J=1.5,0.4 \mathrm{~Hz})$.

3,5- dinitrosalicylyl benzimidazole Ie : White. Yield: $91.3 \%$, m.p. $175-159^{\circ} \mathrm{C}$

${ }^{1} \mathrm{H}$ NMR: $\delta 7.29(1 \mathrm{H}$, ddd, $J=7.5,5.9,1.5 \mathrm{~Hz}), 7.42(1 \mathrm{H}$, ddd, $J=7.5,7.4,1.6 \mathrm{~Hz}), 7.93-$ $8.01(2 \mathrm{H}, 7.98(\mathrm{ddd}, J=5.9,1.6,0.5 \mathrm{~Hz}), 7.96(\mathrm{ddd}, J=7.4,1.5,0.5 \mathrm{~Hz})), 8.70(1 \mathrm{H}, \mathrm{d}, J=$ $1.7 \mathrm{~Hz}), 8.84(1 \mathrm{H}, \mathrm{d}, J=1.7 \mathrm{~Hz})$. 


\section{ACKNOWLEDGEMENTS:}

I thankful to the konkan Gyanpeeth Rahul Dharkar College of Pharmacy and Research Institute, Karjat, M.S. for their support in the work.

\section{REFERENCES:}

1. Gaba, M., Dhingra N., Microwave Chemistry: General Features and Applications, Ind J Pharm Edu Res, 45(2), 2011, 175-183.

2. Khatun S., Khan M.Z.H., Khatun K., Sattar M.A., Microwave-Assisted Synthesis of Arylidene Acetophenones, 2013.

3. Nain S., Singh R., Ravichandran S., Importance of Microwave Heating in Organic Synthesis, Adv J Chem A 2019, 2(2), 94-104.

4. Man A.K., Shahidan R., Microwave-assisted Chemical Reactions, Journal of Macromolecular Science, Part A Pure and Applied Chemistry, 44(6), 2007, 651-657.

5. Shah J.J., Mohanraj K., Comparison of Conventional and Microwave-assisted Synthesis of Benzotriazole Derivatives, Indian J Pharm Sci. 2014 Jan-Feb; 76(1): 4653.

6. Priecel P., Sanchez J.A.L., Advantages and Limitations of Microwave Reactors: From Chemical Synthesis to the Catalytic Valorization of Biobased Chemicals, ACS Sustainable Chem. Eng. 2019, 7, 3-21.

7. Newrekar M.V., Green Synthesis and Antihelminthic Activity of 2-Aryl Benzimidazole Derivatives, IJPPR, 13 (4), 2018

8. Banjare S.K., Payra S., Saha A., Banerjee S., Efficient Room Temperature Synthesis of 2-Aryl benzimidazoles Using ZnO Nanoparticles as Reusable Catalyst, Organic \& Medicinal Chem IJ. 2017; 1(4): 555568.

9. Menteşe, E., Bektaş H., Ülker, S., Bekircan O., Kahveci, B., Microwave-assisted synthesis of new benzimidazole derivatives with lipase inhibition activity, J Enzyme Inhib Med Chem, 2014; 29(1): 64-68.

10. Hasan Kucukbay, PART I: Microwave-Assisted Synthesis of Benzimidazoles: An Overview (Until 2013), Küçükbay, JOTCSA. 2017; 4(1): 1-22.

11. Kathirvelan D, Yuvaraj P, Babu K, Arasampattu S et.al., A green synthesis of Benzimidazoles, IJC, 52B, 2013, 1152-1156.

12. Ramjith, U.S., Banda, G.M., Microwave Assisted Synthesis of Benzimidazole Thiazine Derivatives, IJRPS, 2012,2(3),146-158. 